\title{
Decline in intelligence is associated with progression in white matter hyperintensity volume
}

\author{
E Garde, E Lykke Mortensen, E Rostrup, O B Paulson
}

\begin{abstract}
Objectives: To quantify the time course of white matter hyperintensities (WMH) and assess the association between progression and cognitive decline in non-demented octogenarians.

Methods: From a Danish cohort of 698 people born in 1914, 26 participated in neuropsychological assessment (Wechsler adult intelligence scale) initiated at age 50, including cognitive testing and cerebral magnetic resonance imaging at the 80 and 85 year studies. WMH volumes were quantified and partial correlations were calculated between WMH volume change and decline in WAIS scores from 80 to 85 . Results: Progression in WMH volume ranged from $0 \mathrm{ml}$ to $20.7 \mathrm{ml}$, providing a median increase of $2.6 \mathrm{ml}$ (range 0.1 to $20.7, p<0.001)$ and, with a mean time interval between scans of 3.8 years, a rate of progression of 0.63 (0 to 6.8) $\mathrm{ml} /$ year. WMH volume measures for the two hemispheres were highly correlated $(r=0.95)$ and did not differ significantly. Increase in WMH volume was correlated with a simultaneous decline in verbal IQ $(r=-0.65, p=0.001)$, while baseline WMH was associated with subsequent decline in performance subtests (digit symbol, $r=-0.57, p=0.02$ ). Conclusions: The association between $\mathrm{WMH}$ and decline in essential cognitive abilities even in well preserved elderly people suggests that WMH should be regarded as a risk factor for cognitive impairment and dementia.
\end{abstract}

W hite matter hyperintensities (WMH) are often observed on MRI and known to be highly related to age. ${ }^{1}$ Increasing evidence supports the view that WMH have detectable clinical implications even in healthy elderly subjects. ${ }^{2-7}$ However, while some cross sectional studies have observed cognitive impairment or dementia as an accompaniment of $\mathrm{WMH}^{8-10}$ others have only been able to confirm an association between WMH and previous ${ }^{3}$ or subsequent decline in cognitive function. ${ }^{2}{ }^{411} 12$ Longitudinal studies with serial MRI are still scarce, and although progression of WMH volumes has been detected no association with cognitive decline has been reported. ${ }^{8}{ }^{13}$

So far no study with repeated MRI and cognitive assessment is known to us. In this population based follow up study we prospectively quantified the changes in WMH volume in community dwelling octogenarians. The objective of this study was to describe the temporal relation between changes in WMH volume and decline in intelligence.

\section{METHODS}

The Glostrup 1914 cohort comprises 976 individuals born in 1914 and living in seven municipalities close to Copenhagen County Hospital in Glostrup in $1964 .{ }^{14}$ At the initial 50 year study, 698 individuals participated in the psychological study which was repeated at ages 60, 70, 80, and 85. At each wave, comprehensive data collection and medical evaluations were also conducted. ${ }^{15}$ In addition, at the 80 year assessment, 75 of the 136 individuals, who took part in the psychological follow up and who had participated in all previous studies, agreed to participate in an MRI study. ${ }^{3}$ At the 85 year follow up 48 of these 76 subjects were alive. This study was conducted as several home visits during which the participants were given verbal and written information on the MRI study. Three subjects were excluded because of routine MRI contraindications, two died before the scan, and 17 declined (refusal rate $35 \%$ ). The present sample comprises 26 subjects (54\%) who completed both 80 and 85 year cognitive testing and MRI scanning. Mean (SD) interval between cognitive assessment and MRI examination was $1.7(0.5)$ years at the 80 year study, and $0.2(0.1)$ years at the 85 year study. The study was approved by the Copenhagen County scientific ethics committee (KA99167), and the procedures accorded with the Helsinki Declaration II.

For all cognitive assessments the same Danish translation of the complete WAIS (Wechsler adult intelligence scale) or part of it was used. ${ }^{16} 17$ Although only 15 of the 26 participants completed all WAIS subtests, prorating made it possible to compute a verbal IQ for all participants, and performance and full scale IQs for $23 .{ }^{17} \mathrm{In}$ addition to the WAIS, the mini-mental state examination (MMSE) was applied in the 85 year study to assess global cognitive function. ${ }^{18}$

MRI was carried out on the same 1.5 Vision scanner (Siemens, Erlangen, Germany) and with an identical double spin echo sequence (time of repetition (TR)/time of echo (TE), $2800 / 20$ and $80 \mathrm{~ms}$; matrix $=256 \times 192$; field of view $(\mathrm{FOV})=230 \mathrm{~mm} ; 60$ axial $2 \mathrm{~mm}$ thick slices) provided proton density and T2 weighted images. ${ }^{3} \mathrm{WMH}$ were defined as clearly hyperintense areas relative to surrounding white matter on both proton density and T2 weighted images and identified by simultaneous inspection of both images. Infarcts (areas that were hyperintense on T2 weighted imaging and isointense on proton density but with corresponding hypointensity on Tl weighted images and located in a vascular distribution area) were noted but not included. Local thresholding was applied and WMH volumes for the whole brain and each hemisphere quantified automatically using in-house developed software (available on request from www.drcmr.dk/software). Visual identification was carried out blinded to date of examination by a trained rater.

To assess repeatability 10 subjects were selected randomly for blinded reanalysis. This resulted in a within subject error of $0.14 \mathrm{ml}$ on single volume measurement (that is, an estimated error on volume changes of $0.2 \mathrm{ml}$ ) and an intraclass correlation coefficient (ICC) of 0.99. For comparison with previous results and with frequently used visual rating methods, both baseline and follow up images were rated blind using a modified version of the Scheltens scale, as described in detail elsewhere. ${ }^{3}$ A significant correlation was

Abbreviations: ICC, intraclass correlation coefficient; MMSE, minimental state examination; WAIS, Wechsler adult intelligence scale; WMH, white matter hyperintensity 
Table 1 Clinical characteristics of the study sample

\begin{tabular}{|c|c|c|c|c|}
\hline \multirow[b]{2}{*}{ Variable } & \multicolumn{3}{|l|}{50 year study } & \multirow{2}{*}{$\begin{array}{l}80 \text { year study } \\
\text { MRI sample }\end{array}$} \\
\hline & MRI sample & No MRI sample & $\begin{array}{l}\text { Total study } \\
\text { population }\end{array}$ & \\
\hline Sample size & 26 & 671 & 698 & 26 \\
\hline Age (years) & $50.2(0.1)$ & $50.5(0.3)$ & $50.5(0.2)$ & $80.7(0.4)$ \\
\hline Education (score*) & $4.0(1.5)$ & $3.5(1.4)$ & $3.5(1.4)$ & $4.0(1.5)$ \\
\hline Sex, male & $16(62 \%)$ & $375(56 \%)$ & $391(56 \%)$ & $16(62 \%)$ \\
\hline BP, systolic (mm Hg) & $136.0(17.4)$ & $139.2(20.6)$ & $139.0(20.5)$ & $148.3(20.4)$ \\
\hline BP, diastolic (mm Hg) & $83.7(8.6)$ & $87.3(13.1)$ & $87.2(13.0)$ & $79.9(9.3)$ \\
\hline Blood glucose (mmol/l) & $5.5(0.5)$ & $5.7(1.1)$ & $5.7(1.1)$ & $5.8(2.3)$ \\
\hline BMI $\left(\mathrm{kg} / \mathrm{m}^{2}\right)$ & $23.8(2.4)$ & $25.2(4.0)$ & $25.2(4.0)$ & $25.8(2.9)$ \\
\hline Cholesterol, total (mmol/l) & $7.7(1.2)$ & $7.4(1.3)$ & $7.4(1.3)$ & $6.2(0.9)$ \\
\hline Hypertension, history/treated & $5(19 \%)$ & $176(27 \%)$ & $181(26 \%)$ & $7(27 \%)$ \\
\hline Diabetes, history/treated & $0(0 \%)$ & $12(2 \%)$ & $12(2 \%)$ & $2(8 \%)$ \\
\hline Angina & $4(15 \%)$ & $124(18 \%)$ & $128(18 \%)$ & $4(15 \%)$ \\
\hline \multicolumn{5}{|c|}{$\begin{array}{l}\text { Values are mean (SD) or } n(\%) \text {. } \\
{ }^{*} \mathrm{~A} 7 \text { point index based on a combination of school education (scored on a } 1 \text { to } 3 \text { point scale) and vocational } \\
\text { training (scored on a } 1 \text { to } 5 \text { point scale). } .^{20} \\
\text { BMI, body mass index; BP, blood pressure; MRI, magnetic resonance imaging. }\end{array}$} \\
\hline
\end{tabular}

found between rated score and WMH volume for baseline (Pearson $r=0.75, \mathrm{p}<0.001)$ and follow up values $(r=0.80$, $\mathrm{p}<0.001)$, but not between the corresponding progression values $(r=0.29)$, confirming results from a recent study. ${ }^{19}$

As MRI hyperintensity volumes had a positively skewed distribution, all analyses were repeated using a square root transformation of WMH volumes. In analyses of correlations between WMH volume and WAIS scores, sex, education, and the corresponding WAIS test score at age 50 were included as covariates in an attempt to reduce statistical noise caused by non-age-related individual differences in cognitive function. ${ }^{20}$

\section{RESULTS}

Demographic data for the 26 individuals participating in both MRI studies are shown in table 1. For comparison, data for the MRI sample and total population at age 50 are also shown. Participants who participated in both MRI studies 35 years later had a slightly lower body mass index at age 50 $(p=0.067)$ and were better educated $(p=0.094)$, but no significant differences were found between the groups.
Table 2 presents IQ scores, WMH volumes, and correlations from the regression analysis of WAIS decline scores and WMH volumes. From baseline to follow up a significant decline in test scores was observed for the three WAIS IQs and several subtests. No significant sex difference in decline scores was found. At the 80 year study, the 26 subjects participating in both MRI examinations obtained higher mean IQ scores than the 22 subjects participating in the 85 year psychological study but declining a scan at follow up. However, with a standard deviation for full scale IQs of 16.81 at baseline and 16.12 at follow up for the MRI sample, and a theoretical population standard deviation of WAIS IQs of 15, the sample variance is not restricted to an extent that prohibits analysis of the correlations between MRI results and intellectual functioning. Mean MMSE score at follow up was also significantly higher for the 26 follow up subjects than for the 22 who declined a second MRI, at 26.9 (2.7) $v$ $24.2(5.3), \mathrm{p}=0.03$.

Progression in WMH volume ranged from $0 \mathrm{ml}$ to $20.7 \mathrm{ml}$ (table 2) and with an average follow up period of 3.8 years

Table 2 MRI volumes, WAIS test scores, and correlation between IQ change and WMH measures

\begin{tabular}{|c|c|c|c|c|c|c|c|c|}
\hline \multirow[b]{3}{*}{ Variable } & \multicolumn{3}{|c|}{ Measured MRI and WAIS values } & \multicolumn{5}{|c|}{ Correlations } \\
\hline & \multirow[b]{2}{*}{ Baseline } & \multirow[b]{2}{*}{ Follow up } & \multirow[b]{2}{*}{ Change } & \multirow{2}{*}{$\begin{array}{l}\text { Baseline } \\
\text { adjusted }\end{array}$} & \multirow{2}{*}{$\begin{array}{l}\text { Follow up } \\
\text { adjusted }\end{array}$} & \multicolumn{3}{|l|}{ Increase } \\
\hline & & & & & & Adjusted & Non-adjusted & $\mathrm{n}$ \\
\hline WMH volume/ml & 4.7 (0 to 44.2$)$ & $9.3(0.1$ to 49.3$)$ & ) $2.6(0.1 \text { to } 20.7)^{*}$ & & & & & \\
\hline Full IQ & $95.52(16.12)$ & $90.39(16.81)$ & $-5.13(7.81) \dagger$ & -0.23 & $-0.40 \S$ & $-0.43 \S$ & -0.35 & 23 \\
\hline Verbal IQ & $97.73(16.68)$ & $93.04(16.54)$ & $-4.69(8.46) \dagger$ & -0.28 & $-0.52 \dagger$ & $-0.65^{*}$ & $-0.53 \dagger$ & 26 \\
\hline Information & $19.62(4.04)$ & $18.00(4.53)$ & $-1.62(2.37) \dagger$ & -0.12 & -0.29 & $-0.47 \ddagger$ & $-0.45 \ddagger$ & 26 \\
\hline Comprehension & $15.58(4.27)$ & $15.50(4.51)$ & $-0.08(2.68)$ & $-0.45 \ddagger$ & $-0.55 \dagger$ & -0.35 & -0.35 & 26 \\
\hline Arithmetic & $11.00(3.23)$ & $10.25(3.19)$ & $-0.75(1.92)$ & -0.33 & -0.42 & -0.38 & -0.34 & 20 \\
\hline Similarities & $17.23(4.60)$ & $16.00(4.96)$ & $-1.23(3.48)$ & -0.15 & -0.30 & $-0.40 \S$ & -0.27 & 26 \\
\hline Digit span & $9.38(1.88)$ & $9.19(1.79)$ & $-0.19(1.65)$ & -0.08 & -0.29 & $-0.52 \dagger$ & $-0.41 \ddagger$ & 26 \\
\hline Vocabulary & $56.58(10.53)$ & $51.88(12.66)$ & $-4.69(5.92)^{*}$ & 0.02 & -0.05 & -0.16 & 0.03 & 26 \\
\hline Performance IQ & $93.30(13.28)$ & $88.09(15.05)$ & $-5.22(9.18) \ddagger$ & -0.06 & -0.08 & -0.06 & -0.01 & 23 \\
\hline Digit symbol & $34.38(9.80)$ & $29.43(9.95)$ & $-4.95(5.45)^{*}$ & $-0.57 \dagger$ & $-0.44 \S$ & -0.11 & -0.01 & 21 \\
\hline Picture completion & $12.39(3.61)$ & $11.83(3.26)$ & $-0.57(2.74)$ & 0.07 & -0.02 & -0.18 & -0.19 & 23 \\
\hline Block design & $30.64(5.69)$ & $28.77(6.27)$ & $-1.86(4.16) \pm$ & 0.09 & 0.09 & 0.03 & 0.07 & 22 \\
\hline Picture arrangement & $18.45(6.48)$ & $15.80(6.01)$ & $-2.65(4.37) \ddagger$ & -0.21 & -0.01 & 0.37 & 0.13 & 20 \\
\hline Object assembly & $28.68(6.29)$ & $27.47(7.69)$ & $-1.21(6.80)$ & -0.20 & -0.13 & 0.08 & 0.08 & 19 \\
\hline
\end{tabular}

WMH volumes are median (range) and WAIS raw scores are mean (SD).

For WMH increase, the table presents adjusted (partial) and non-adjusted (bivariate) correlations with cognitive decline between the two assessments. Partial correlations are adjusted for sex, education, and the corresponding 50 year test score; $\mathrm{n}$ number of subjects completing the test. ${ }^{*} \mathrm{p}<0.001 ; \mathrm{tp}<0.01 ; \neq \mathrm{p}<0.05 ; \S \mathrm{p}<0.1$.

MRI, magnetic resonance imaging; WAIS, Wechsler adult intelligence scale; WMH, white matter hyperintensities. 
(range 3.0 to 4.8 years) amounted to a median rate of progression of 0.63 (0 to 6.8$) \mathrm{ml}$ per year. WMH volume measures for the two hemispheres were highly correlated $(r=0.95, \mathrm{p}<0.001)$ and did not differ significantly. Baseline MRI showed lacunar infarcts in five subjects but no cortical infarcts. At follow up one more participant had a lacunar infarct while two subjects revealed clinically undetected cortical infarcts.

Increase in WMH volume was significantly correlated to a simultaneous decline in verbal IQ $(r=-0.65, \mathrm{p}=0.001)$, and baseline WMH was associated with subsequent decline in performance subtests (digit symbol $r=-0.57, \mathrm{p}=0.02$ ) but no association was observed between WMH volume and absolute WAIS or MMSE test scores. The correlations were similar for both hemispheres. Adjusting for the 80 year test score instead of the 50 year score had no marked effect on the partial correlations and, when baseline WMH was included as a covariate, the analyses showed essentially the same results as reported here. Analysis based on a square root transformation of WMH volume showed a very similar pattern of results.

\section{DISCUSSION}

Our most remarkable finding is the significant correlation between increase in WMH volume and a simultaneous decline in verbal IQ scores.

Previously published data from our 80 year study of the 1914 cohort show substantial decline in the verbal part of the WAIS, although still less than the often observed decline in non-verbal subtests. ${ }^{20}$ The verbal tests mainly assess accumulated knowledge and experience and the ability to access this material. In daily life, impairment in these functions probably plays an even more important role than impairment of non-verbal functions. Bearing in mind the small sample size, a correlation of 0.65 - corresponding to $43 \%$ explained variance-suggests strong effects of WMH increase in old age.

Although smaller than most cross sectional studies, our study benefits from a community dwelling sample, homogeneous with respect to age. As in most longitudinal studies, selection bias is unavoidable, and may have weakened the insample relation between increase in WMH volume and cognitive decline. If so, the true association has been underestimated. On the other hand, both WMH volume and cognitive performance scores varied considerably in our population and, with frequencies of cardiovascular risk factors similar to those reported in other population studies, they suggest susceptibility to lifelong exposure to cardiovascular risk factors. ${ }^{4}{ }^{6}$ Accordingly, our sample does not seem highly selected despite the unavoidable effect of selective mortality.

As our subjects have participated in this population study for 35 years, baseline assessment of cognitive performance could be included in the correlation analysis. We regard the test scores from age 50 as the best available proxy for the large individual differences in cognitive function before the onset of age related changes. Such pre-existing individual differences may dilute the correlation between WMH and cognitive function. Consequently, inclusion of the corresponding 50 year test score as a covariate should lead to higher partial correlations. This is indeed what we observed.

Another interesting finding is the significant correlation between baseline WMH volumes and subsequent decline in cognitive function. This corroborates previous findings and supports the view that WMH may be a valuable predictor of clinical outcome. ${ }^{11}{ }^{12}$ Larger longitudinal studies are needed, but our results suggest that the presence of WMH should be taken seriously, irrespective of the size of the WMH and the age of patient. We suggest that WMH be regarded a risk factor which may be associated with decline in essential cognitive abilities, even in well preserved older subjects, within a relatively short time span.

\section{ACKNOWLEDGEMENTS}

We thank the MRI technicians and other staff involved in data collection at the Danish Research Centre of Magnetic Resonance (Hvidovre), and the Centre of Preventive Medicine (Glostrup). We acknowledge the contribution of Mogens Kleven (deceased) to all psychological studies of the 1914 cohort.

This research was supported by the Velux Foundation, the Copenhagen Hospital Corporation Research Foundation, the Elsass Foundation, and the Simon Spies Foundation. The sponsors had no role in study design, data collection, data analysis, data interpretation, or writing the report.

\section{Authors' affiliations}

E Garde, E Rostrup, Danish Research Centre for Magnetic Resonance, Copenhagen University Hospital, Hvidovre, Denmark

E Lykke Mortensen, Department of Health Psychology, Institute of Public Health, University of Copenhagen, Copenhagen, Denmark

O B Paulson, Neurobiology Research Unit, Copenhagen University Hospital, Rigshospitalet, Denmark

Competing interests: none declared

Correspondence to: Dr Ellen Garde, National Hospital for Neurology and Neurosurgery, Dementia Research Centre, Box 16, 8-11 Queen

Square, London WCIN 3BG, UK; elleng@magnet.drcmr.dk

Received 8 October 2004

In revised form 30 January 2005

Accepted 31 January 2005

\section{REFERENCES}

1 Christiansen $\mathbf{P}$, Larsson HB, Thomsen $C$, et al. Age dependent white matter lesions and brain volume changes in healthy volunteers. Acta Radiol 1994:35: 117-22.

2 DeCarli C, Miller BL, Swan GE, et al. Cerebrovascular and brain morphologic correlates of mild cognitive impairment in the National Heart, Lung, and Blood Institute twin study. Arch Neurol 2001;58:643-7

3 Garde E, Mortensen EL, Krabbe K, et al. Relation between age-related decline in intelligence and cerebral white-matter hyperintensities in healthy octogenarians: a longitudinal study. Lancet 2000;356:628-34.

4 de Groot JC, de Leeuw FE, Oudkerk M, et al. Periventricular cerebral white matter lesions predict rate of cognitive decline. Ann Neurol 2002;52:335-41.

5 Gunning-Dixon FM, Raz N. Neuroanatomical correlates of selected executive functions in middle-aged and older adults: a prospective MRI study. Neuropsychologia 2003;41:1929-41.

6 Koga H, Yuzuriha T, Yao H, et al. Quantitative MRI findings and cognitive impairment among community dwelling elderly subjects. J Neurol Neurosurg Psychiatry 2002;72:737-41.

7 Leaper SA, Murray AD, Lemmon HA, et al. Neuropsychologic correlates of brain white matter lesions depicted on MR images: 1921 Aberdeen Birth Cohort. Radiology 2001;221:51-5.

8 Cook IA, Leuchter AF, Morgan ML, et al. Longitudinal progression of subclinical structural brain disease in normal aging. Am J Geriatr Psychiatry 2004; 12:190-200.

9 Schmidt R, Enzinger C, Ropele S, et al. Progression of cerebral white matter lesions: 6-year results of the Austrian Stroke Prevention Study. Lancet 2003:361:2046-8.

10 Soderlund H, Nyberg L, Adolfsson R, et al. High prevalence of white matter hyperintensities in normal aging: relation to blood pressure and cognition. Cortex 2003:39:1093-105.

11 Kuller LH, Lopez OL, Newman A, et al. Risk factors for dementia in the cardiovascular health cognition study. Neuroepidemiology 2003;22:13-22.

12 Wu CC, Mungas D, Petkov Cl, et al. Brain structure and cognition in a community sample of elderly Latinos. Neurology 2002;59:383-91.

13 Whitman GT, Tang Y, Lin A, et al. A prospective study of cerebral white matter abnormalities in older people with gait dysfunction. Neurology 2001:57:990-4.

14 Hagerup L, Hansen PF. 50-aars undersoegelsen i Glostrup. Ugeskr Laeger 1968;130:1145-8.

15 Korsgaard TK, Larsen S, Schroll M. Cardiovascular risk factors and age: a cross-sectional survey of Danish men and women from the Glostrup population studies, 1991. Am J Geriatr Cardiol 1995;4:31-41.

16 Wechsler D. Wechsler adult intelligence scale manual. New York: Psychological Corporation, 1955.

17 Mortensen EL, Kleven M. A WAIS longitudinal study of cognitive development during the life span from ages 50 to 70. Dev Neuropsychol 1993;9:115-30.

18 Folstein MF, Folstein SE, McHugh PR. "Mini-mental state". A practical method for grading the cognitive state of patients for the clinician. J Psychiatr Res 1975; 12:189-98.

19 Prins ND, van Straaten EC, van Dijk EJ, et al. Measuring progression of cerebral white matter lesions on MRI: visual rating and volumetrics. Neurology 2004;62:1533-9.

20 Mortensen E, Hogh P. A gender difference in the association between APOE genotype and age-related cognitive decline. Neurology 2001;57:89-95. 